\title{
Impacts of roads and trails on floral diversity and structure of Ganga-Choti forest in Kashmir Himalayas
}

\author{
Impactos de caminos y senderos en la diversidad de flora y la estructura \\ del bosque de Ganga-Choti en Cachemira, Himalaya
}

\author{
Hamayun Shaheen ${ }^{a *}$, Amra Riffat a, Maria Salik ${ }^{a}$, Syeda Sadiqa Firdous a \\ ${ }^{*}$ Corresponding author: ${ }^{a}$ University of Azad Jammu and Kashmir, Department of Botany, \\ Muzaffarabad, 13100 Pakistan, phone: +92-344-5952552, hamayunmaldial@yahoo.com
}

\begin{abstract}
SUMMARY
Roads and trails bring deteriorating changes in the structure and composition of forests. The present study investigated the impact of roads and trails on floral diversity and structure of Ganga Choti forest, Kashmir. Phytosociological attributes using systematic quadrate sampling were recorded and analyzed using multivariate ordination techniques including Principal Component Analysis and Cluster Analysis. The total number of species found in the area was 101 belonging to 47 plant families. The dominant family was Poaceae followed by Labiateae, Asteraceae and Rosaceae. The dominant species of the area included Pinus wallichiana, Abies pindrow, Viburnum grandiflorum, Sarcococca saligna, Poa alpina, Fragaria nubicola, Onychium japonicum and Poa pratensis. The dominant life form was Hemicryptophyte (31.68 \%), whereas leaf spectra class was Microphyll (30.69 \%). The average diversity value was 3.39 with a maximum of 3.71 at the control site and 3.08 at the disturbed site. Average tree density was 505/ha with a maximum of $680 /$ ha at the control site and a minimum of 330/ha at the disturbed site. Intense deforestation was indicated by a stump density value of 330/ha. Results revealed that roads and trails have adversely affected the composition and structure of vegetation with high pressure on keystone tree species. Disturbed sites showed lower values of diversity indices, species richness and regeneration synchronized with high deforestation, overgrazing, erosion and increased frequency of invasive species. The study area needs immediate attention of the management for conservation of local forests by minimizing the impacts of roads and trails.
\end{abstract}

Key words: deforestation, Himalayas, species diversity, overgrazing.

\section{RESUMEN}

Los caminos y senderos deterioran la estructura y composición de los bosques. El presente estudio investigó el impacto de los caminos y senderos en la diversidad de flora y la estructura del bosque de Ganga Choti, Cachemira. Los atributos fitosociológicos se registraron mediante muestreo cuadrado sistemático. Los atributos fitosociológicos se registraron utilizando un muestreo cuadrado sistemático y se analizaron mediante técnicas de ordenación multivariante, que incluyeron análisis de componentes principales y análisis de conglomerados. Se encontraron 101 especies plantas en el área, pertenencias a 47 familias. La familia dominante fue Poaceae seguida de Labiateae, Asteraceae y Rosaceae. Las especies dominantes del área incluyeron Pinus wallichiana, Abies pindrow, Viburnum grandiflorum, Sarcococca saligna, Poa alpina, Fragaria nubicola, Onychium japonicum y Poa pratensis. La forma de vida dominante fue hemicriptófita $(31,68 \%)$, mientras que la clase de espectros de hojas fue microfila $(30,69 \%)$. El valor de diversidad promedio fue 3,39 con un máximo de 3,71 en el sitio de control y 3,08 en el sitio alterado. La densidad promedio de árboles por hectárea fue de 505 con un máximo de 680 en el sitio de control y un mínimo de 330 en el sitio alterado. La deforestación intensa fue indicada por un valor de densidad de tocones por hectárea de 330. Los resultados revelaron que los caminos y senderos han afectado adversamente la composición y la estructura de la vegetación con alta presión sobre especies clave de árboles. Los sitios alterados mostraron valores menores de índices de diversidad, riqueza de especies y regeneración, sincronizados con alta deforestación, pastoreo excesivo, erosión y mayor frecuencia de especies invasoras. El área de estudio necesita una atención inmediata de la administración para la conservación de los bosques locales para minimizar los impactos de los caminos y senderos.

Palabras clave: deforestación, Himalaya, diversidad de especies, sobrepastoreo.

\section{INTRODUCTION}

Forest paths and trails strongly influence plant species composition in natural forests (Godefroid and Koedam 2004). Road infrastructure, and the access that it provides, degrades forest ecosystem by increasing edge habitats, reducing forest cover, increasing agricultural expansion, promoting migration and forestland encroachment (Verburg et al. 2004). Roads affect forest ecosystem by altering the level of available light, water, drainage patterns, 
soil structure and soil nutrients, and act as a barrier to migration and dispersal (van-Wyk and Smith 2001). Major impact on forest ecosystem regarding function is due to alteration of microclimate and the isolation of ecosystem patches by causing fragmentation and creating edges (Hill and Pickering 2006).

Road constructions also affect the impact on conservation potential of the area with secondary impacts such as people influx, economic development and construction activities. The invasion of alien weeds and invasive plants is another major issue associated with road development (Hua et al. 2013). Roads are the major source of landslides and sediment loss as well (Pietikainen 2006). Flow of surface and ground water and nutrients is affected by trails changing the geomorphic processes and sediment loads. Roads provide an easy passageway to nomads as livestock rearing has been the main source of livelihood in Himalayas for centuries (Kumar et al. 2001). Effects of trampling may exceed up to one meter from the trail edge by causing compaction of leaf litter and soil, reducing the abundance of woody and herbaceous plants (Nilsson 2001).

Population rise has resulted in exceeding forest use beyond the carrying capacity of the forest ecosystem in Kashmir Himalayas. Roads and trails are the easy passageways for the population to access the forest area for their needs, resulting into forest degradation (Shameem et al. 2010). The study area has four link roads and numerous trails used for the movement of locals and their livestock. The current study is based on the hypothesis that roads and trails have significant deteriorating consequences on forest structure and species diversity in the study area. Furthermore, roads and trails are associated with increased anthropogenic forest disturbances such as logging, over- grazing and erosion affecting floral diversity and forest structure. The specific objective of the study is to investigate the effects of roads and trails on floral diversity, species composition, richness, community maturity and evenness of forest stands. It was further aimed at analyzing the impact of anthropogenic pressure regarding deforestation, overgrazing and soil degradation on the phytosociological attributes of forest vegetation.

\section{METHODS}

The study area lies in Azad Jammu and Kashmir in Pir Panjal Sub range of western Himalayas. Sudhan Galli is located at an elevation of $2300 \mathrm{~m}$ at $34^{\circ} 04^{\prime} 34.92^{\prime \prime} \mathrm{N}$ latitude and $73^{\circ} 44^{\prime} 54.52^{\prime \prime}$ E Longitude (figure 1). Topographically the entire area is mountainous, generally sloping from northeast to southwest covered with moist temperate forest vegetation. Annual precipitation is about 1,500 millimeter. May and June are the hottest months with average temperature of $20-25^{\circ} \mathrm{C}$, while December and January are the coldest months with heavy snowfall and freezing temperature (Pak-Met 2014). The study was carried out during May-August 2016. Four forest sites were selected for the study including two disturbed and two control sites. The altitude, latitude and longitude of all study sites were recorded. The effect of roads and trails on vegetation distribution was investigated by placing quadrates along trails and roads. The quadrats were laid 5-20 $\mathrm{m}$ away from the trails and 5-50 $\mathrm{m}$ away from the roads. The quadrate size of $1 \mathrm{~m} \times 1 \mathrm{~m}$ was used for the herbs, whereas for trees and shrubs quadrats size was $20 \mathrm{~m} \times 20 \mathrm{~m}$ and $5 \mathrm{~m} \times 5 \mathrm{~m}$ respectively. Vegetation attributes were recorded including density, frequency and cover. Phytosociological attributes

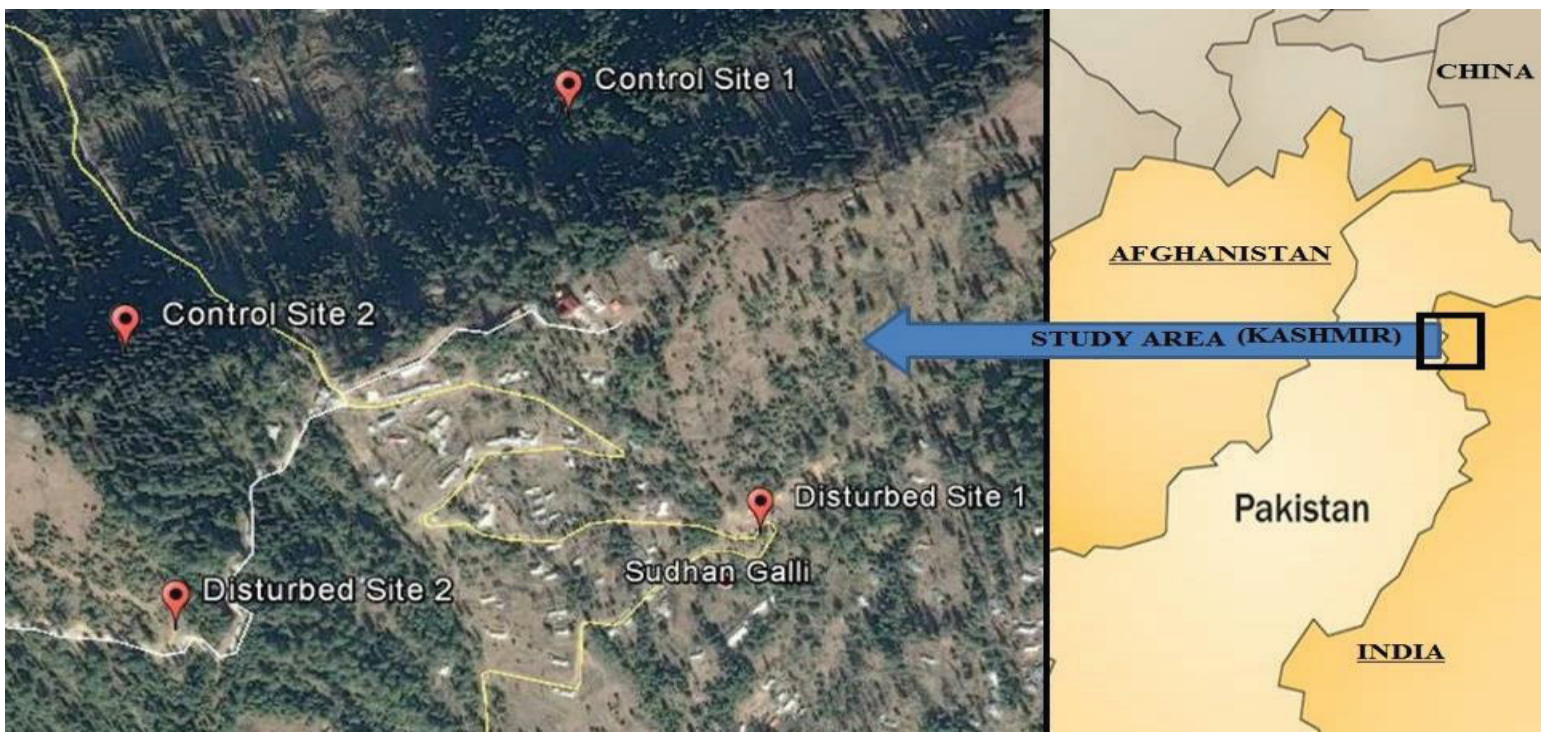

Figure 1. Map of the study area (Right) and satellite imagery of study sites (Left).

Mapa del área de estudio (derecha) e imágenes satelitales de los sitios de estudio (izquierda). 
including Importance Value Index, Diversity, Evenness, Richness and maturity were calculated following standard protocols (Cox 1967, Ahmed and Shaukat 2012). Visual indicators such as hoof marks, animal excreta, browsed vegetation, eroded area and signs of trampling were used to determine grazing intensity. Deforestation rate was recorded by counting the number of stumps, whereas regeneration rate was calculated by counting the number of seedlings (Shaheen et al. 2011). Results were statistically analyzed by using Multivariate ordination techniques including Principal Component Analysis and Cluster Analysis (ter-Braak and Smilaur 1998).

\section{RESULTS}

A total of 101 plants were recorded from the study area belonging to 46 families. The dominant family was Poaceae with 11 species followed by Lamiaceae or Labiateae, Asteraceae and Rosaceae with nine members each. Pteridaceae displayed five species, whereas Pinaceae and Polygonaceae showed four species each. The dominant species of the area included Pinus wallichiana and Abies pindrow with IVI values of 79.44 and 67.9, respectively, followed by Viburnum grandiflorum (36.83), Sarcococca saligna (19.8), Poa alpina, Fragaria nubicola, Onychium japonicum, Poa pratensis and Oxalis corniculata. Hemicryptophytes was the dominated life form class having a percentage of 31.68 followed by Phanerophytes (18.8\%) and Geophytes (16.8\%). Microphylls were the dominant leaf spectrum class with a percentage of 30.69 followed by Mesophyll (28.7\%) and Nanophylls (26.7\%) (table 1).

The number of species was higher (65) in the control sites range as compared to disturbed sites (43). Density, frequency and cover values recorded for the disturbed sites were also lower than those of control sites (table 2). The Average Shannon diversity value in the area was 3.39 with a maximum of 3.89 recorded at control site 2 , whereas a minimum of 2.93 at disturbed site 1 . The disturbed sites showed lower values (0.97) as compared with the control sites (3.71). Simpson diversity was calculated to be 0.97 . Average evenness was 0.86 with a maximum of 0.94 at control site 2 , whereas a minimum of 0.78 at dis-

Table 1. Floristic composition, importance values, life form and leaf spectra of studied sites.

Composición florística, valores de importancia, forma de vida y espectros foliares en los sitios estudiados.

\begin{tabular}{|c|c|c|c|c|c|}
\hline & Species Name & Family & $\begin{array}{l}\text { Life } \\
\text { form }\end{array}$ & $\begin{array}{c}\text { Leaf } \\
\text { spectrum }\end{array}$ & $\begin{array}{l}\text { Importance } \\
\text { value }\end{array}$ \\
\hline 1 & Abies pindrow Royle & Pinaceae & MP & $\mathrm{L}$ & 44.88 \\
\hline 2 & Achillea millefolium $\mathrm{L}$. & Asteraceae & $\mathrm{H}$ & $\mathrm{L}$ & 0.70 \\
\hline 3 & Adiantum venustum D. Don & Adiantaceae & G & $\mathrm{L}$ & 1.47 \\
\hline 4 & Aesculus indica Wall. Ex Camb. & Hippocastinaceae & MP & $\mathrm{Me}$ & 1.98 \\
\hline 5 & Ajuga bracteosa Wall. ex Benth. & Lamiaceae & $\mathrm{TH}$ & Mi & 0.50 \\
\hline 6 & Arisaema flavum (Forsk.) Schott. & Araceae & G & $\mathrm{Me}$ & 2.10 \\
\hline 7 & Arisaema jacquemontii Blume & Araceae & $\mathrm{G}$ & $\mathrm{Me}$ & 1.25 \\
\hline 8 & Aristolochia punjabensis Lace & Aristolochiaceae & $\mathrm{L}$ & $\mathrm{Mi}$ & 0.96 \\
\hline 9 & Artemisia dubia Wall. ex Besser & Asteraceae & $\mathrm{H}$ & $\mathrm{N}$ & 0.10 \\
\hline 10 & Arthroxon prinodes (Steud.) & Poaceae & $\mathrm{H}$ & $\mathrm{N}$ & 2.92 \\
\hline 11 & Asplenium adiantum-nigrum $\mathrm{L}$. & Adiantaceae & G & $\mathrm{L}$ & 1.28 \\
\hline 12 & Aster himalaicus C.B. Clarke & Asteraceae & $\mathrm{H}$ & Mi & 0.30 \\
\hline 13 & Berberis lycium Royle. & Berberidaceae & NP & $\mathrm{N}$ & 2.54 \\
\hline 14 & Bergenia ciliata (Haw.) Sternb. & Saxifragaceae & $\mathrm{H}$ & $\mathrm{Me}$ & 0.73 \\
\hline 15 & Bistorta amplexicaulis (D. Don) & Polygonaceae & $\mathrm{H}$ & Mi & 3.45 \\
\hline 16 & Bupleurum falcatum Linn. & Umbelliferae & $\mathrm{TH}$ & $\mathrm{N}$ & 0.66 \\
\hline 17 & Calamintha umbrosa Fisch. et Meyer & Lamiaceae & $\mathrm{H}$ & $\mathrm{Mi}$ & 1.46 \\
\hline 18 & Caltha alba Jacq.ex.Camb. & Ranunculaceae & $\mathrm{CH}$ & $\mathrm{Me}$ & 0.15 \\
\hline 19 & Caltha palustris L. & Ranunculaceae & $\mathrm{CH}$ & $\mathrm{Me}$ & 1.21 \\
\hline 20 & Cedrus deodara (Roxb. ex D. Don) & Pinaceae & MP & $\mathrm{L}$ & 0.35 \\
\hline
\end{tabular}


Table 1 Continued

\begin{tabular}{|c|c|c|c|c|c|}
\hline 21 & Chrysopogon echinulatus (Nees) & Poaceae & $\mathrm{H}$ & $\mathrm{N}$ & 0.20 \\
\hline 22 & Cichorium intybus $\mathrm{L}$. & Asteraceae & $\mathrm{CH}$ & $\mathrm{Me}$ & 0.25 \\
\hline 23 & Cirsium vulgare (Savi) Ten. & Asteraceae & TH & $\mathrm{Ma}$ & 4.79 \\
\hline 24 & Convolvulus arvensis $\mathrm{L}$. & Convolvulaceae & $\mathrm{L}$ & $\mathrm{Me}$ & 0.18 \\
\hline 25 & Conyza bonariensis $\mathrm{L}$. & Asteraceae & $\mathrm{TH}$ & Mi & 0.92 \\
\hline 26 & Cotoneaster rosea Edgew. & Rosaceae & NP & $\mathrm{L}$ & 0.32 \\
\hline 27 & Cynodon dactylon $\mathrm{L}$. & Poaceae & $\mathrm{H}$ & $\mathrm{N}$ & 1.28 \\
\hline 28 & Cynoglossum glochidiatum Wall. ex. Benth. & Boraginaceae & $\mathrm{TH}$ & Mi & 1.21 \\
\hline 29 & Cyperus compressus $\mathrm{L}$. & Cyperaceae & G & $\mathrm{N}$ & 0.23 \\
\hline 30 & Dactylis glomerata L. & Poaceae & $\mathrm{H}$ & $\mathrm{N}$ & 2.79 \\
\hline 31 & Desmodium elegans DC. & Papilionaceae & NP & $\mathrm{Me}$ & 1.20 \\
\hline 32 & Digitaria sanguinalis (Linn.) & Poaceae & $\mathrm{H}$ & $\mathrm{N}$ & 0.84 \\
\hline 33 & Dryopteris juxtaposita Christ. & Pteridaceae & $\mathrm{H}$ & $\mathrm{N}$ & 0.23 \\
\hline 34 & Dryopteris stewartii More & Pteridaceae & G & $\mathrm{Me}$ & 4.48 \\
\hline 35 & Duchesnea indica (Andrews) & Rosaceae & $\mathrm{H}$ & Mi & 3.53 \\
\hline 36 & Elaeagnus umbellata Thunb. & Elaeagnaceae & NP & Mi & 0.47 \\
\hline 37 & Equisetum arvense $\mathrm{L}$. & Equisetaceae & $\mathrm{TH}$ & $\mathrm{N}$ & 0.47 \\
\hline 38 & Erigeron multiradiatus Benth. ex C. B. Clarke & Asteraceae & $\mathrm{TH}$ & Mi & 0.40 \\
\hline 39 & Euphorbia helioscopia L. & Euphorbiaceae & $\mathrm{CH}$ & Mi & 0.19 \\
\hline 40 & Fragaria nubicola Lindl. & Rosaceae & $\mathrm{H}$ & Mi & 9.46 \\
\hline 41 & Galium aparine $\mathrm{L}$. & Rubiaceae & $\mathrm{TH}$ & $\mathrm{N}$ & 3.72 \\
\hline 42 & Gentiana kurroo Royle & Gentianaceae & $\mathrm{TH}$ & $\mathrm{N}$ & 0.89 \\
\hline 43 & Geranium wallichianum D. Don. ex. Sweet. & Geraniaceae & $\mathrm{H}$ & Mi & 3.61 \\
\hline 44 & Hedera helix auct. & Araliaceae & $\mathrm{L}$ & $\mathrm{Me}$ & 1.58 \\
\hline 45 & Hedera himalaica Tobler & Araliaceae & $\mathrm{L}$ & $\mathrm{Me}$ & 0.58 \\
\hline 46 & Impatiens bicolor $\mathrm{L}$. & Balsaminaceae & G & $\mathrm{Me}$ & 4.11 \\
\hline 47 & Impatiens edgeworthii Hook. f., Fl. Brit. Ind. & Balsaminaceae & G & $\mathrm{Me}$ & 1.58 \\
\hline 48 & Indigofera heterantha Wall.ex Brandi & Papilionaceae & NP & $\mathrm{N}$ & 1.91 \\
\hline 49 & Isodon rugosus Wall. ex Benth. & Lamiaceae & $\mathrm{MC}$ & $\mathrm{Me}$ & 2.88 \\
\hline 50 & Jasminum humile L. & Oleaceae & $\mathrm{H}$ & $\mathrm{N}$ & 2.29 \\
\hline 51 & Lilium polyphyllum D. Don & Liliaceae & $\mathrm{G}$ & Mi & 0.38 \\
\hline 52 & Lonicera quinquelocularis Hardwicke & Caprifoliaceae & $\mathrm{MC}$ & $\mathrm{Me}$ & 1.80 \\
\hline 53 & Machilus odoratissima Wall. ex Nees & Lauraceae & MP & $\mathrm{Me}$ & 2.34 \\
\hline 54 & Micromeria biflora Benth & Lamiaceae & $\mathrm{TH}$ & $\mathrm{L}$ & 1.00 \\
\hline 55 & Nepeta erecta Boyle ex Benth. & Lamiaceae & $\mathrm{CH}$ & Mi & 4.89 \\
\hline 56 & Oenothera rosea L Her. Ex Ait & Onagraceae & $\mathrm{CH}$ & Mi & 0.86 \\
\hline 57 & Onychium japonicum Thunb. & Pteridaceae & G & $\mathrm{L}$ & 5.83 \\
\hline 58 & Origanum vulgare $\mathrm{L}$. & Lamiaceae & $\mathrm{H}$ & Mi & 0.77 \\
\hline 59 & Oxalis corniculata $\mathrm{L}$. & Oxalidaceae & $\mathrm{H}$ & $\mathrm{N}$ & 4.32 \\
\hline 60 & Phalaris paradoxa Lion. & Poaceae & TH & $\mathrm{N}$ & 1.30 \\
\hline 61 & Picea smithiana (Wall.) Boiss & Pinaceae & MP & $\mathrm{L}$ & 2.77 \\
\hline
\end{tabular}


Table 1 Continued

\begin{tabular}{|c|c|c|c|c|c|}
\hline 62 & Pinus wallichiana A B. Jackson & Pinaceae & MP & $\mathrm{L}$ & 45.66 \\
\hline 63 & Plantago lanceolata $\mathrm{L}$. & Plantaginaceae & $\mathrm{H}$ & Mi & 1.43 \\
\hline 64 & Plantago major $\mathrm{L}$. & Plantaginaceae & $\mathrm{H}$ & $\mathrm{Me}$ & 3.88 \\
\hline 65 & Poa alpina $\mathrm{L}$. & Poaceae & $\mathrm{H}$ & $\mathrm{N}$ & 7.55 \\
\hline 66 & Poa pratensis $\mathrm{L}$. & Poaceae & $\mathrm{H}$ & $\mathrm{N}$ & 3.48 \\
\hline 67 & Polygonum aviculare $\mathrm{L}$. & Polygonaceae & $\mathrm{H}$ & $\mathrm{L}$ & 3.73 \\
\hline 68 & Polygonum nepalense Meisner. & Polygonaceae & G & $\mathrm{Me}$ & 0.87 \\
\hline 69 & Potentilla anserina $\mathrm{L}$. & Rosaceae & $\mathrm{H}$ & $\mathrm{N}$ & 0.85 \\
\hline 70 & Prunella vulgaris $\mathrm{L}$. & Lamiaceae & $\mathrm{TH}$ & Mi & 2.61 \\
\hline 71 & Prunus padus L. & Rosaceae & MP & $\mathrm{Me}$ & 2.31 \\
\hline 72 & Pteris cretica $\mathrm{L}$. & Pteridaceae & $\mathrm{G}$ & $\mathrm{Mi}$ & 2.29 \\
\hline 73 & Pteris vittata $\mathrm{L}$. & Pteridaceae & G & Mi & 1.40 \\
\hline 74 & Quercus incana Roxb. & Fagaceae & MP & $\mathrm{Me}$ & 2.81 \\
\hline 75 & Ranunculus muricatus $\mathrm{L}$. & Ranunculaceae & $\mathrm{CH}$ & $\mathrm{Me}$ & 2.62 \\
\hline 76 & Rosa macrophylla Lindl. & Rosaceae & $\mathrm{L}$ & Mi & 0.57 \\
\hline 77 & Rubus arcticus $\mathrm{L}$. & Rosaceae & $\mathrm{Np}$ & Mi & 0.20 \\
\hline 78 & Rubus fruticosus $\mathrm{L}$. & Rosaceae & $\mathrm{Np}$ & Mi & 2.06 \\
\hline 79 & Rumex nepalensis Spreng. & Polygonaceae & $\mathrm{CH}$ & Mi & 4.95 \\
\hline 80 & Salvia lanata Roxb. & Lamiaceae & $\mathrm{CH}$ & $\mathrm{Me}$ & 0.41 \\
\hline 81 & Sambucus wightiana Wall.ex Wight et Arn. & Sambucaceae & $\mathrm{CH}$ & $\mathrm{Me}$ & 0.65 \\
\hline 82 & Sarcococca saligna (D.Don) Muell. Arg. & Buxaceae & NP & $\mathrm{Me}$ & 7.46 \\
\hline 83 & Sauromatum venosum (Ait.) Schott. & Araceae & G & $\mathrm{Me}$ & 0.31 \\
\hline 84 & Sedum ewersii Ledeb. & Crassulaceae & G & Mi & 0.15 \\
\hline 85 & Sibbaldia cunneata O.kunz & Rosaceae & $\mathrm{H}$ & $\mathrm{N}$ & 0.68 \\
\hline 86 & Sisymbrium irio L. & Brassicaceae & $\mathrm{TH}$ & Mi & 0.44 \\
\hline 87 & Skimmia laureola DC & Rutaceae & $\mathrm{NP}$ & $\mathrm{Me}$ & 2.67 \\
\hline 88 & Solanum nigrum L. & Solanaceae & $\mathrm{CH}$ & Ma & 0.56 \\
\hline 89 & Stellaria media $\mathrm{L}$. & Caryophyllaceae & $\mathrm{H}$ & $\mathrm{N}$ & 2.13 \\
\hline 90 & Tagetes minuta $\mathrm{L}$. & Asteraceae & $\mathrm{TH}$ & $\mathrm{N}$ & 0.19 \\
\hline 91 & Taraxacum officinale Weber & Asteraceae & $\mathrm{H}$ & Mi & 2.29 \\
\hline 92 & Themeda anathera (Nees) Hack. & Poaceae & $\mathrm{H}$ & $\mathrm{N}$ & 0.19 \\
\hline 93 & Thymus linearis Benth. & Lamiaceae & $\mathrm{CH}$ & Mi & 1.36 \\
\hline 94 & Trifolium repens $\mathrm{L}$. & Poaceae & $\mathrm{H}$ & $\mathrm{N}$ & 8.67 \\
\hline 95 & Urtica dioica $\mathrm{L}$. & Urticaceae & $\mathrm{H}$ & $\mathrm{Me}$ & 2.46 \\
\hline 96 & Valeriana pyrolifolia Decne. & Valerianaceae & G & Mi & 0.20 \\
\hline 97 & Verbascum thapsus $\mathrm{L}$. & Scrophulariaceae & $\mathrm{TH}$ & Ma & 0.63 \\
\hline 98 & Viburnum grandiflorum Wall. ex DC & Caprifoliaceae & NP & $\mathrm{Me}$ & 19.74 \\
\hline 99 & Viola canescens Wall. ex.Roxb. & Violaceae & G & Mi & 5.76 \\
\hline 100 & Viola odorata $\mathrm{L}$. & Violaceae & $\mathrm{H}$ & Mi & 0.96 \\
\hline 101 & Vulfia myrosus L. & Poaceae & $\mathrm{H}$ & $\mathrm{N}$ & 0.88 \\
\hline
\end{tabular}

Life form. MP: Megaphanerophytes= $7.69 \%$. MC: Microphanerophytes $=1.92 \%$. NP: Nanophanerophytes $=10.57 \%$. CH: Chamaephytes $=12.5 \%$. TH: Therophytes $=14.42 \%$. G: Geophytes $=17.30 \%$. L: Liana $=4.807 \%$. H: Hemicryptophytes $=30.7 \%$.

Leaf spectra. Ma: Macrophyll $=2.88 \%$. Me: Mesophyll $=28.84 \%$. Mi: Microphyll $=30.76 \%$. L: Leptophyll $=11.53 \%$. N: Nanophyll $=25.96 \%$. 
turbed site 1. Average richness recorded for the area was 1.81 with a maximum richness value of 2.23 at disturbed site 1 , whereas a minimum value of 1.48 at disturbed site 2 . The disturbed sites showed lower values of maturity index (25.75) when compared with 34.6 at control sites. The average maturity value recorded from the area was 30.17 with a minimum of 23.13 at disturbed site 1 , whereas a maximum of 42.22 at control site 2 . None of the forest sites was found to be mature $(>60 \%)$ as the average maturity index values for the investigated area was 30.18. Relatively higher values were reported at control sites (34.6) than those reported for disturbed sites (25.75) revealing the negative impact of roads and trails on the forest (table 2).

Forest stands showed tree density of 505/ha with a maximum of 760 /ha at control site 1 , whereas a minimum of 200/ha at disturbed site 2. The disturbed sites showed lower density values (330/ha) as compared with 680/ha at control sites. Intense deforestation and logging were indicated at the disturbed sites with a stump density value of 520/ha as compared with the 150/ha at control sites. Average stump density recorded from the area was 335/ha with maximum stump density of 600/ha at disturbed site 2, whereas a minimum of 100/ha at control site 2. Average stem/stump value recorded from the area was 2.78 with a maximum value of 6 at control site 2 , whereas a minimum value of 0.3 at disturbed site 2 . Regeneration value in the area was 290 seedling/ha with a maximum of 500/ha at control site 2 , whereas a minimum of $0 /$ ha at disturbed site 2 . The disturbed sites showed lower regeneration rate (130/ha), compared with 430/ha at control sites (table 3).

Disturbed sites showed higher grazing intensity placed in grazing classes 2 and 3 showing maximum hoof marks, animal excreta and browsed vegetation. Control sites showed relatively lower grazing pressure. Disturbed site 1 was highly eroded and placed in class 3 , while disturbed site 2 was moderately eroded and placed in class 2 , within erosion classes. No visible effect of erosion was observed at the control sites.

PCA axis explained $94 \%$ variance in the data indicating the statistical strength of the test. PCA Biplot showed dominant species of the area separated away along the Xaxis; i.e., Pinus wallichiana and Abies pindrow representing their ultra-dominance. Viburnum grandiflorum, Poa

Table 2. Phytosociological parameters of forest communities from the study area.

Parámetros fitosociológicos de las comunidades forestales del área de estudio.

\begin{tabular}{lcccccc}
\hline \multicolumn{1}{c}{ Study Site } & $\begin{array}{c}\text { Specie } \\
\text { number }\end{array}$ & $\begin{array}{c}\text { Simpson } \\
\text { diversity }\end{array}$ & $\begin{array}{c}\text { Shannon } \\
\text { diversity }\end{array}$ & Richness & Evenness & $\begin{array}{c}\text { Maturity } \\
\text { Index }\end{array}$ \\
\hline Control 1 & 68 & 0.94 & 3.53 & 1.86 & 0.84 & 26.98 \\
Control 2 & 63 & 0.98 & 3.89 & 1.66 & 0.94 & 42.22 \\
Average control & 65.5 & 0.96 & 3.71 & 1.76 & 0.89 & 34.60 \\
Disturbed 1 & 43 & 0.99 & 2.93 & 2.23 & 0.78 & 23.13 \\
Disturbed 2 & 40 & 0.97 & 3.22 & 1.48 & 0.87 & 28.37 \\
Average disturbed & 41.5 & 0.98 & 3.08 & 1.86 & 0.83 & 25.75 \\
Overall average & 53.5 & 0.97 & 3.39 & 1.81 & 0.86 & 30.17 \\
\hline
\end{tabular}

Table 3. Structural attributes and disturbance indicators of studied forest stands.

Atributos estructurales e indicadores de perturbación de rodales forestales estudiados.

\begin{tabular}{|c|c|c|c|c|c|c|}
\hline Study site & $\begin{array}{c}\text { Tree } \\
\text { density/ha }\end{array}$ & $\begin{array}{c}\text { Stump } \\
\text { density/ha }\end{array}$ & $\begin{array}{c}\text { Stem/ } \\
\text { stumps value }\end{array}$ & Seedlings/ha & $\begin{array}{l}\text { Erosion } \\
\text { class }\end{array}$ & $\begin{array}{c}\text { Grazing } \\
\text { class }\end{array}$ \\
\hline Control 1 & 760 & 200 & 3.8 & 400 & class 1 & class 1 \\
\hline Control 2 & 600 & 100 & 6.0 & 500 & class 1 & class 1 \\
\hline Average control & 680 & 150 & 4.9 & 450 & un-eroded & ungrazed \\
\hline Disturbed 1 & 460 & 440 & 1.04 & 260 & class 3 & class 2 \\
\hline Disturbed 2 & 200 & 600 & 0.3 & 0 & class 2 & class 3 \\
\hline Average disturbed & 330 & 520 & 0.67 & 130 & eroded & grazed \\
\hline Overall average & 505 & 335 & 2.78 & 290 & & \\
\hline
\end{tabular}


alpina and Trifolium repens were significantly correlated with the disturbed sites. Cirsium vulgare, Nepeta erecta, Polygonum aviculare, Rumex nepalensis, Poa pratensis, Urtica dioica, Onychium japonicum, Skimmia laureola, Bistorta amplexicaulis were also found clumped near the disturbed sites. On the other hand, Fragaria nubicola, Sarcococca saligna, Dactylis glomerata and Stellaria media showed strong correlation with the control site (figure 2). The cluster analysis dendrogram revealed 104 species arranged in five major associations grouped based on correlation and dominance in communities. Abies pindrow, Pinus wallichiana and Viburnum grandiflorum were branched out at the1st cut level, having maximum relative abundance at all sites. The $2^{\text {nd }}$ association comprised 9 co-dominant species, whereas the $3^{\text {rd }}$ association had 24 species abundantly found at disturbed sites. The species of control sites were clustered in the $4^{\text {th }}$ and $5^{\text {th }}$ groups with 24 and 15 species respectively (figure 3 ).

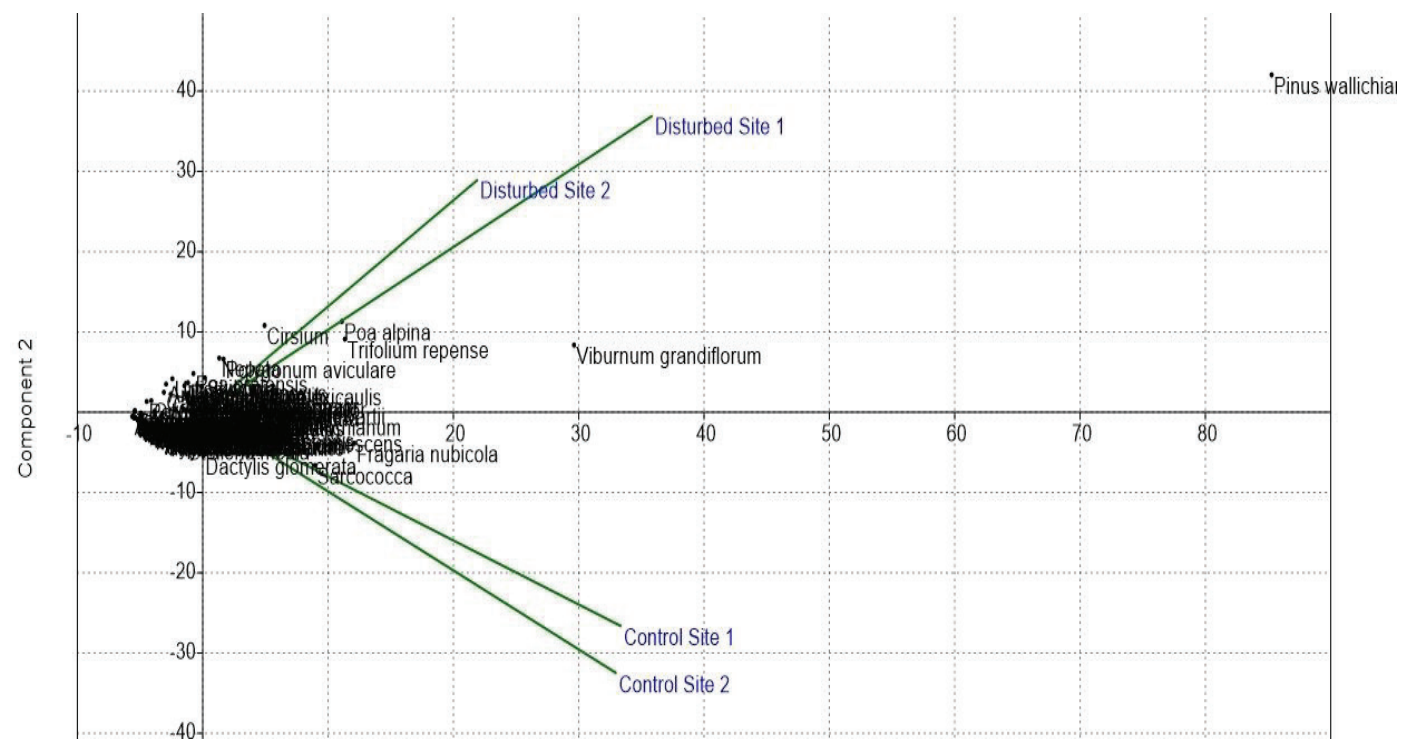

Figure 2. Principal Component Analysis Biplot of the species and study sites.

Gráfico del análisis de componentes principales de las especies y sitios de estudio.

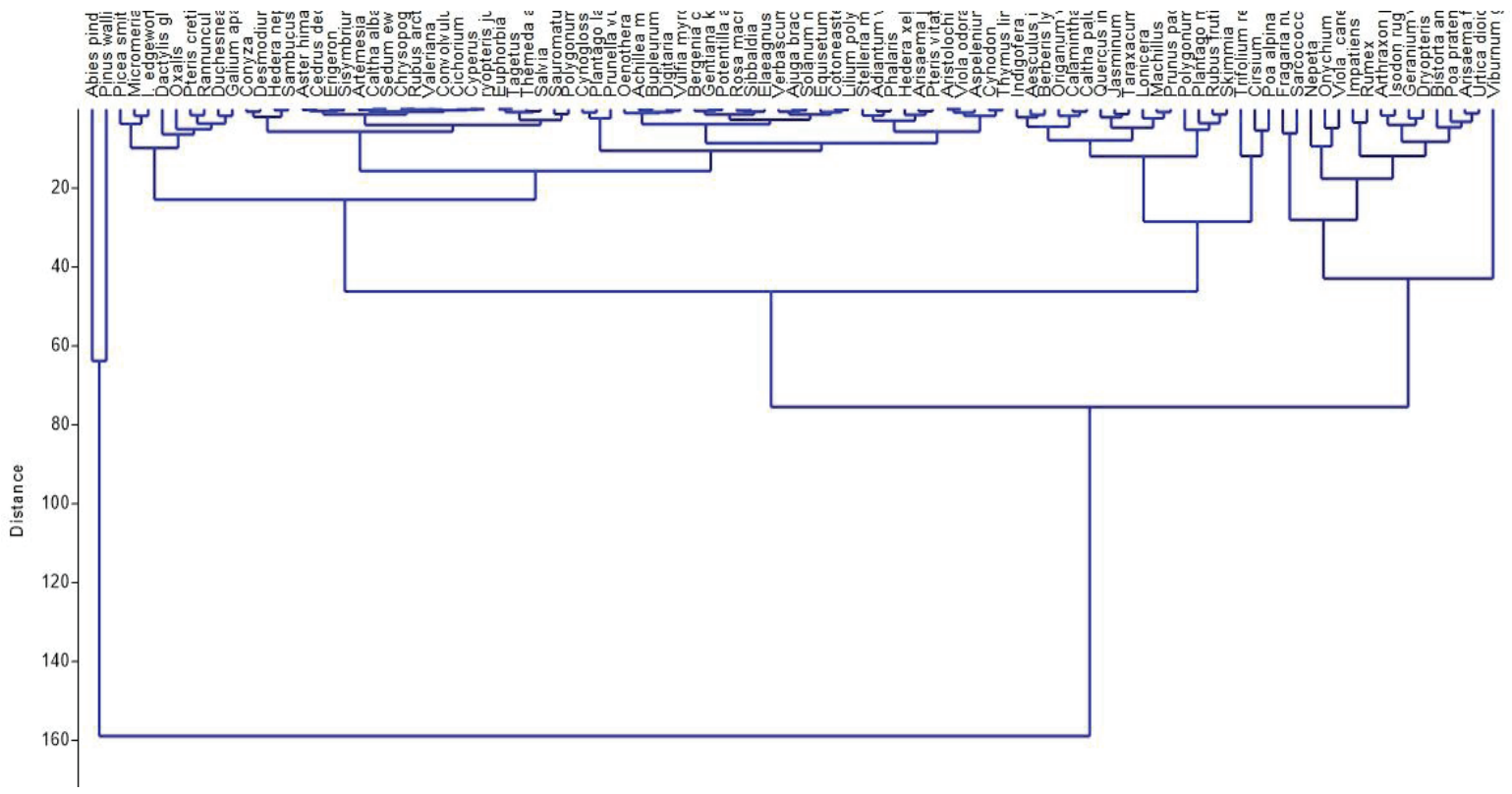

Figure 3. Cluster Analysis Dendrogram of the species data based on Euclidean distance.

Dendrograma del análisis de conglomerados de los datos de especies basado en la distancia euclidiana. 


\section{DISCUSSION}

The current study was based on the assessment of impact of roads and trails on the vegetation of the Ganga Choti forests. The forest structure and composition of the investigated area showed important influence of the impacts of roads and trails. Vegetation parameters fluctuated significantly correlated with the intensity of road and trail disturbance.

Average tree density of the forest stands was calculated to be $505 /$ ha. This value is less than 1828 /ha calculated in the temperate forest of Garhwal Himalaya (Devlal and Sharma 2008), 610/ha in Subtropical Zone of Garhwal Himalaya, India (Tiwari et al. 2010), 1028/ha in the temperate forest of Garhwal Himalaya (Kumar et al. 2001), 963/ha in the temperate forest of Arunachal Pradesh, India (Bharali et al. 2011), 820/ha in Gangotri valley, India (Dhaulkhandi et al. 2008) and 602/ha in Himalayas of Pakistan (Ahmed et al. 2006). Low tree density indicates high disturbance due to fuelwood and timber extraction at disturbed areas (Sagar et al. 2003). This is synchronized with high stump density $(520 / \mathrm{ha})$ at disturbed sites indicating severe deforestation and pressure. Presence of roads and trails are as low as $1 \%$ of the land area and can degrade the vegetation of an area up to $20 \%$ (Nepstad et al. 2001). Locals access the forest for fuel wood, timber, fodder and non-timber products leading to forest degradation.

Natural regeneration was as poorly represented as 130 seedlings/ha at disturbed sites. Average regeneration rate calculated for the studied area was 290 seedlings/ha, which is less than 5,474/ha in the Sikkim Himalaya (Sundriyal et al. 1994), 5,200/ha in Gangotri, India (Dhaulkhandi et al. 2008) and 2681/ha in Garhwal Himalaya, India (Tiwari et al. 2010). High disturbance prevents the vegetation from reaching the climax community. Simpson's diversity value of 0.98 showed the instability of vegetation communities at the investigated sites due to high intensity of anthropogenic pressure, microclimatic variations and edaphic factors correlated with roads and trails disturbance (Sen et al. 2008).

Average value of specie richness was high at the disturbed site (1.86) as compared with 1.76 at control sites, which showed the invasion of non-native flora at the site. The new land use type favors the non-native species and non-managed alien vegetation (Cardoso et al. 2013). Mild disturbances and high moisture level increase the species richness allowing increased light, water and nutrients in open trail sections as compared with closed tree canopies of control plots (Atik et al. 2011). The percentage of climbers/lianas was very low $(4.9 \%)$ in the area, indirectly indicating the lower trees diversity and density (Dhaulkhandi et al. 2008).

Results revealed high soil erosion at disturbed sites due to road cuttings, exposing the soil to slides and erosion. The forest areas with roads are reported to have fivefold increase in landslide intensity, which is also reflected in our study area (Ovando 2008). Slope is also a key factor influencing potential for impacts to soil and vegetation. Exposed trail slopes are subjected to surface erosion, evident from diminished vegetation cover and reduced plant heights along the trail (White et al. 2006). Roads and trails associated activities cause loss of herbaceous understory and compaction of soil resulting in decreased forest productivity and sediment loss leading to loss of freshwater habitats and altered stream hydrology (Pietikainen 2006).

\section{CONCLUSIONS}

The investigated forest showed significant deteriorating impacts on forest structure and composition associated with the presence of roads and trails. The disturbed sites with roads and trails were characterized by lower floral diversity, species richness and immature communities. High grazing pressure, deforestation intensity and soil degradation were observed at the disturbed sites correlated with roads and trails presence. There is an immediate need for sustainable management of local forests by minimizing the impacts of roads and trails. It is recommended to incorporate biodiversity conservation into the design and implementation of road projects along with Sound road engineering and design work, minimizing forest fragmentation, maintaining partial tree canopy cover and controlling erosion and runoff.

\section{REFERENCES}

Ahmed M, S Shaukat. 2012. A Text book of Vegetation Ecology. Near New Urdu Bazar Karachi, Pakistan. Abrar Sons. 430 p.

Ahmed M, T Hussain, AH Sheikh, SS Hussain, MF Siddiqui. 2006. Phytosociology and structure of Himalayan Forests from different climatic zones of Pakistan. Pakistan Journal of Botany 38(2): 361-382.

Atik M, S Sayan, O Karaguzel, E Yildirim. 2011. Trail Impact Management Related to Vegetation Response in Termessos National Park. In Tiefenbacher J ed. Perspectives on Nature Conservation - Patterns, Pressures and Prospects. Rijeka, Croatia. Intech. p. 223-240.

Bharali S, P Ashish, LK Mohamed, LB Singha. 2011. Species Diversity and Community Structure of a Temperate Mixed Rhododendron Forest along an Altitudinal Gradient in West Siang District of Arunachal Pradesh, India. Nature Science 9(12): 125-140.

Cardoso P, F Rigal, S Fattorini, S Terzopoulou, PAV Borges. 2013. Integrating Landscape Disturbance and Indicator Species in Conservation Studies. Journal Pone 8(5): 63294.

Cox WG. 1967. Laboratory manual of general ecology. Dubuque, IA, USA. WMC Brown. 297 p.

Devlal R, N Sharma. 2008. Altitudinal changes in dominancediversity and species richness of tree species in a temperate forest of Garhwal Himalaya. Life Sciences Journal 5(2): 53-57.

Dhaulkhandi M, A Dobhal, S Bhatt, M Kumar. 2008. Community structure and regeneration potential of natural forest site 
in Gangotri, India. Journal of Basic and Applied Sciences 4(1): 49-52.

Godefroid S, N Koedam. 2004. The impact of forest paths upon adjacent vegetation: Effects of the path surfacing material on the species composition and soil compaction. Biological Conservation 119(3): 405-419.

Hill W, CM Pickering. 2006. Vegetation associated with different walking track types in the Kosciuszko alpine area, Australia. Journal of Environmental Management 78: 24-34.

Hua ZZ, HU Gang, NI Jian. 2013. Effects of Topographical and Edaphic Factors on the Distribution of Plant Communities in two Subtropical Karst Forests, Southwestern China. Journal of Mountain Sciences 10(7): 95-104.

Kumar M, CM Sharma, GS Rajwar, A Mishra. 2001. Community structure and plant biodiversity in relation to disturbance gradient in temperate forest of Garhwal Himalaya. Van Vigyan 39(1-4): 1-9.

Nepstad D, G Carvalho, AC Barros, A Alencar, JP Capobianco, J Bishop, P Moutinho, P Lefebvre, ULS Jr, E Prins. 2001. Road paving, fire regime feedbacks and the future of Amazon forests. Forest Ecology and Management 154(3): 395-407.

Nilsson T. 2001. Management of Communal Grazing Land. A case study on institutions for collective action in Endabeg village, Tanzania. Thesis. Master of Science. Stockholm, Sweden. Royal Institute of Technology. 88 p.

Ovando DAC. 2008. Road impact on deforestation and jaguar habitat loss in the Mayan forest. Thesis. Doctor of Philosophy. England, UK. University Program in Ecology, Graduate School, Duke University. 103 p.

Pak-Met. 2014 The Normals of climatic data of Azad Jammu \& Kashmir. Islamabad, Pakistan. Report. Pakistan Meteorological Department. 9 p.

Pietikainen V. 2006. Measures to prevent overstocking and overgrazing in woodlands. Thesis. Master in scvience. Stochholm, Sweden. School of Life Sciences, Environment and Development Educational Program, Sodertorn University College. 22 p.
Sagar R, AS Raghubanshi, JS Singh. 2003. Tree species composition, dispersion and diversity along a disturbance gradient in a dry tropical forest region of India. Forest Ecology and Management 186: 61-71.

Sen A, T Johri, NS Bisht. 2008. Analysis of the effects of anthropogenic interference on tree species composition in the forests of Dadra and Nagar Haveli, India. Current Science 95:50-58.

Shaheen H, RA Qureshi, Z Ullah, T Ahmad. 2011. Anthropogenic pressure on the western Himalayan moist temperate forests of Bagh, Azad Jammu \& Kashmir. Pakistan Journal of Botany 43(1): 695-703.

Shameem SA, P Soni, G Bhat. 2010. Comparative study of herb layer diversity in lower Dachigam National Park, Kashmir Himalaya, India. International Journal of Biodiversity Conservation 2(10): 308-315.

Sundriyal RC, E Sharma, LK Rai, SC Rai. 1994. Tree Structure, Regeneration and Woody Biomass Removal in a Sub-Tropical Forest of Mamlay Watershed in the Sikkim Himalaya. Vegetatio 113(1): 53-63.

Ter Braak CJF, P Smilauer. 1998. CANOCO Reference Manual and User's Guide to CANOCO for Windows: Software for Canonical Community Ordination (version 4), Microcomputer Power. New York, USA.

Tiwari GB, KI Tadele, FI Aramde, C Tiwari. 2010. Community Structure and Regeneration Potential of Shorea robusta Forest in Subtropical Submontane Zone of Garhwal Himalaya, India. Nature Science 8(1): 70-74.

Van-Wyk AE, G Smith. 2001. Regions of Floristic Endemism in southern Africa. A review with emphasis on succulents. Pretoria, South Africa. Umdaus Press. 199 p.

Verburg PH, KP Overmars. 2004. Accessibility and land-use patterns at the forest fringe in the northwestern part of the Philippines. The Geographical Journal 170(3): 238-255.

White DD, MT Waskey, GP Brodehl, PE Foti. 2006. A comparative study of impacts to mountain bike trails in five common ecological regions of the Southwestern U.S. Journal of Park and Recreation Administration 24(2): 21-41. 
